\title{
ВмJ Global Health Identification and inclusion of gender factors in retrospective cohort studies: the GOING-FWD framework
}

\author{
Valeria Raparelli, ${ }^{1,2}$ Colleen M. Norris, ${ }^{2}$ Uri Bender, ${ }^{3}$ Maria Trinidad Herrero, ${ }^{4}$ \\ Alexandra Kautzky-Willer, ${ }^{5}$ Karolina Kublickiene, ${ }^{6,7}$ Khaled El Emam, ${ }^{8,9,10}$ \\ Louise Pilote, ${ }^{3,11}$ GOING-FWD Collaborators
}

To cite: Raparelli V, Norris CM, Bender $\mathrm{U}$, et al. Identification and inclusion of gender factors in retrospective cohort studies: the GOING-FWD framework. BMJ Global Health 2021;6:e005413. doi:10.1136/ bmjgh-2021-005413

Handling editor Seye Abimbola

Received 16 February 2021 Revised 10 March 2021 Accepted 13 March 2021
Check for updates

C Author(s) (or their employer(s)) 2021. Re-use permitted under CC BY-NC. No commercial re-use. See rights and permissions. Published by BMJ.

For numbered affiliations see end of article.

Correspondence to Dr Louise Pilote; louise.pilote@mcgill.ca

\section{ABSTRACT}

Gender refers to the socially constructed roles, behaviours, expressions and identities of girls, women, boys, men and gender diverse people. Gender-related factors are seldom assessed as determinants of health outcomes, despite their powerful contribution. The Gender Outcomes INternational Group: to Further Well-being Development (GOING-FWD) project developed a standard five-step methodology applicable to retrospectively identify genderrelated factors and assess their relationship to outcomes across selected cohorts of non-communicable chronic diseases from Austria, Canada, Spain, Sweden. Step 1 (identification of gender-related variables): Based on the gender framework of the Women Health Research Network (ie, identity, role, relations and institutionalised gender), and available literature for a certain disease, an optimal 'wish-list' of gender-related variables was created and discussed by experts. Step 2 (definition of outcomes): Data dictionaries were screened for clinical and patient-relevant outcomes, using the International Consortium for Health Outcome Measurement framework. Step 3 (building of feasible final list): a cross-validation between variables per database and the 'wish-list' was performed. Step 4 (retrospective data harmonisation): The harmonisation potential of variables was evaluated. Step 5 (definition of data structure and analysis): The following analytic strategies were identified: (1) local analysis of data not transferable followed by a meta-analysis combining study-level estimates; (2) centrally performed federated analysis of data, with the individual-level participant data remaining on local servers; (3) synthesising the data locally and performing a pooled analysis on the synthetic data and (4) central analysis of pooled transferable data. The application of the GOING-FWD multistep approach can help guide investigators to analyse gender and its impact on outcomes in previously collected data.

\section{INTRODUCTION}

The distinction between sex and gender, which is clear and common in social sciences, has largely been neglected in health sciences. Indeed, sex and gender are often erroneously used and/or measured interchangeably. Given that sex and gender are not
Summary box

The Gender Outcomes INternational Group: to Further Well-being Development (GOING-FWD) framework is a feasible 5-step methodology to assess the impact of gender domains on clinical and patient-relevant outcomes in retrospective studies, allowing a crosscountries comparison.

- A multidisciplinary international team (ie, specialists in life science, social science and computer science; patient partners) built the methodology, guaranteeing that the interests of all stakeholders be (were?) represented.

- The lack of a standardised definition of gender and data accessibility/protection issues are expected obstacles to the applicability of GOING-FWD methodology.

- Strategies to minimise potential drawbacks of the methodology are provided such as the derived 'wish list' of gender-related factors or the application of privacy-enhancing technologies including tools for federated analysis.

independent of each other, solely assessing one or the other cannot account for identified variations in health. ${ }^{12}$ Furthermore, although the reasons explaining the increasing incidence of chronic diseases are incompletely understood, changing family, social, institutional roles and attitudes of men and women in the last decades ultimately play a role. Thus, a wide range of behavioural factors, psychosocial processes, personal, cultural and societal factors can create, suppress or amplify underlying biological health differences. ${ }^{3}$ While differences in health status and outcomes have been attributed to biological sex, it is now increasingly recognised that both sex and gender influence the risk of developing certain diseases, presentation of symptoms, severity of illness, response to drugs or nonpharmacological interventions and seeking care behaviours. ${ }^{5}$ More importantly, gender 
may also have a bearing on people's access to and uptake of health services and the resulting health outcomes experienced throughout the life-course ${ }^{6}$ Consequently, it is now understood that the intersectionality of gender with other social factors such as race, age, ethnicity, culture and sexual orientation, plays a central role in an individual's health. The integration of a gender-based framework in health research is a crucial and long-awaited development. $^{7}$

When considering gender aspects in the evaluation of clinical outcomes, the first challenge for scientists originates from the apparent lack of standardised method to measure the complexities that gender encompasses. Recently, through a Pan-Canadian collaboration of a multidisciplinary team of scientists, a comprehensive list of gender-related variables was established and collected in the setting of premature cardiovascular disease. Constructed with the aim of exploring the impact of gender on the clinical outcomes of young patients with acute coronary syndrome, the Gender and Sex Determinants of Cardiovascular Disease: From Bench to Beyond Gender Score (GPGS) was developed. ${ }^{8}$ The GPGS measures a comprehensive group of genderrelated factors and offers a pragmatic means to prospectively explore the relationships between sex, gender and health outcomes. In patients with premature and established cardiovascular disease, gender factors, independent of biological sex, emerged as powerful predictors of the acquisition of risk factors as well as of 1-year adverse health outcomes. ${ }^{910}$ Most significantly, regardless of sex, patients who exhibited gender factors most traditionally ascribed to women's identity and roles in society were more likely to have a recurrent cardiac event within the first year. While these results have important direct implications for expanding the measurement of gender determinants of health to other populations, they may also identify novel determinants of healthcare costs that could be averted.

To facilitate the integration of sex and gender-based analyses, we developed a standard methodology that can be applied to retrospective studies for testing the associations of gender-related factors with clinical and patientrelevant outcomes.

\section{ABOUT THE GENDER OUTCOMES INTERNATIONAL GROUP: TO FURTHER WELL-BEING DEVELOPMENT (GOING-FWD) INTEGRATION OF GENDER DIMENSIONS INTO HEALTH OUTCOMES RESEARCH}

The Gender Outcomes INternational Group: to Further Well-being Development (GOING-FWD) is a personalised medicine project that explores the effect of sex and gender on outcomes across already available datasets using feasible approaches to perform both traditional and machine-learning-based analytics. It was recently cofunded by the Canadian Institutes of Health Research, and GENDER-NET plus, which is a part of the European
EU H2020 initiative (http://gender-net-plus.eu/jointcall/funded-projects/going-fwd/).

For the GOING-FWD project, around thirty accessible databases of observational studies and registries that include non-communicable chronic diseases (NCDs) among a four-country transatlantic network (ie, Austria, Canada, Spain and Sweden) were identified. The overarching aims of the GOING-FWD project were (1) to integrate sex and gender dimensions in applied health research and (2) to evaluate their impact on clinical cost-sensitive outcomes and patient-reported outcomes related to quality of life in NCDs including cardiovascular disease, metabolic disease, chronic kidney disease (CKD) and neurodegenerative disease. Each partner of the Consortium provided the data dictionary of the retrospective cohort studies conducted in their respective countries.

The GOING-FWD Consortium is composed of investigators with multidisciplinary expertise in gender dimension, psychosocial science, computer science, epidemiology, endocrinology, internal medicine, renal and cardiovascular medicine, reproductive health, neuroscience, preclinical and clinical experimental research, health outcome research, nursing and biostatistics. The investigators were assigned to one of the three-work packages. The GOING-FWD methodology proposed therein is the result of the integrated activities carried out by the GOING-FWD investigators from March 2019 to December 2019. A five-step procedure was developed that can be applied to pre-existing observational cohorts for the integration of gender-related factors in assessing their association with selected health outcomes.

GOING-FWD also has a patient partner advocate group. All interactions with patient partners are based on inclusiveness, support, mutual respect and cobuilding. For example, patient partners can assist in knowledge dissemination (eg, summer institutes, online educational materials, trainee journal club meetings, public forum presentations, may coauthor manuscripts and provide feedback on draft manuscripts during development and participate in teleconferences. A patient partner representative also attends monthly GOING-FWD steering meetings.

\section{THE GOING-FWD ROADMAP FOR ALREADY COLLECTED DATA}

A multistep methodology was developed as summarised in figure 1 .

\section{Step 1: identification of gender-related variables}

Based on the data dictionaries provided by all participating centres, a preliminary list of the gender-related factors available in selected datasets was compiled by the coordinating centre. The template including the identified sex and gendered factors was presented and discussed at the first consortium meeting (Montréal, April 2019) by all investigators and stakeholders. Guided by the gender framework of the Women Health Research 


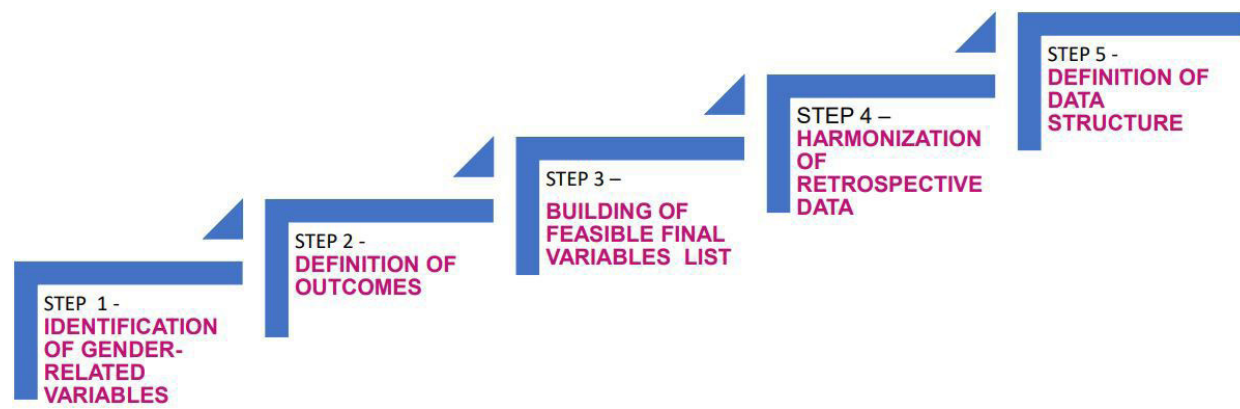

Figure 1 The GOING-FWD multistep methodology on identification and inclusion of gender factors in retrospective cohort studies. GOING-FWD, Gender Outcomes INternational Group: to Further Well-being Development.

Network (ie, gender identity, gender role, gender relations and institutionalised gender) ${ }^{11}$ (figure 2), and available literature in the four NCDs areas, the investigators created an optimal 'wish list' of gender-related variables/ factors: the definitions and validity of the proposed variables were discussed and expert consensus reached.

Investigators considered variables that differ between men and women in terms of prevalence and/or identified (in the published literature) as exerting different effects on the outcomes of men and women as 'gender-related' variables. A revised draft template including additional gendered variables was created (table 1).

\section{Step 2: definition of outcomes}

Each of the cohort data dictionaries was screened for outcomes of interest (including clinical/survival and patient-reported outcomes) by the coordinating centre. Similar to gendered variables, a second working group was tasked with developing a list of outcome variables, using the International Consortium for Health Outcome Measurement (ICHOM) framework $^{12}$ [The ICHOM Standard Sets are standardised outcomes, measurement tools, time points and risk adjustment factors for a given condition (eg, CKD, diabetes, etc). Developed by a consortium of experts and patients in the field of outcomes research, the ICHOM Standard Sets focus on clinical and patient-centred outcomes. By creating a standardised list of the outcomes based on the patient's priorities, the ICHOM framework ensures that the patient remains at the centre of care] and cost-sensitive variables and/or patient-reported outcome measures (PROMs) collected in all databases as the basic 'outcomes variable list'. A prespecified list of potential outcomes was created by all GOING-FWD investigators.

Depending on the study population and type of dataset (eg, administrative, observational cohort study),

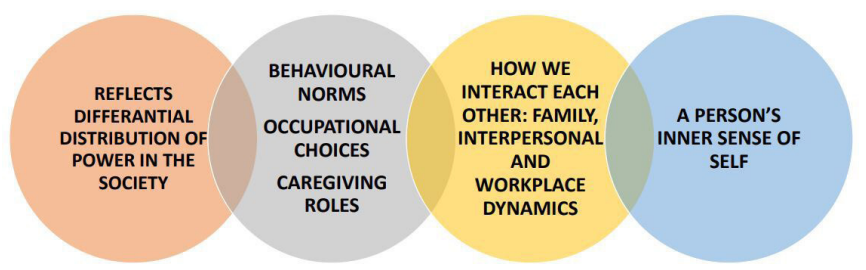

Figure 2 Domains that gender encompasses. we identified as relevant the following cost-sensitive outcomes: (1) inpatient outcomes including: in-hospital length of stay, in-hospital complications and/or death and readmission within 30 days of discharge; (2) outpatient outcomes including: access and/or numbers of visits and procedures, admissions, death, progression of disease and disability. We also looked for the availability of any PROMs, including symptoms (eg, pain), functioning, health related quality of life, depression and stress. The ICHOM specific-disease outcomes were considered for each of the four main clinical areas of interest. The revised draft template with outcomes, compiled by the investigators, was discussed and approved by all (table 2).

\section{Step 3: building of feasible final list}

The two lists were sent to each participating centre to rescreen their datasets for the presence of the identified sex- and gender-related as well as outcome variables. A cross-validation between gender-related and outcomes variables available per database was performed both locally and centrally. In case of disagreement or discordant definitions of variables among the wish-list and the actuallist, a discussion to reach consensus between coordinator centre and local principal investigators was performed. In principle, a more inclusive approach was pursued for both gender-related variables and outcomes definition.

After the double check of wish-list and local actual-list, the final feasible list of variables (core dictionary) was built, and each country partner used the lists to apply to their respective research ethics boards according to the country regulations.

\section{Step 4: retrospective data harmonisation}

Once the final list was compiled, the harmonisation potential of gender-related and outcomes variables was assessed using the Maelström Research guidelines for rigorous retrospective data harmonisation and merging when possible ${ }^{13}$ (Core Dataset).

The harmonisation across the different databases is a premise for assessing the feasibility of big data analysis, as well as minimising deviations in data measurement across independently recruited databases. Data harmonisation methodology consists of assessing the presence and definitions of common variables across the different databases, followed by the creation of a harmonised 


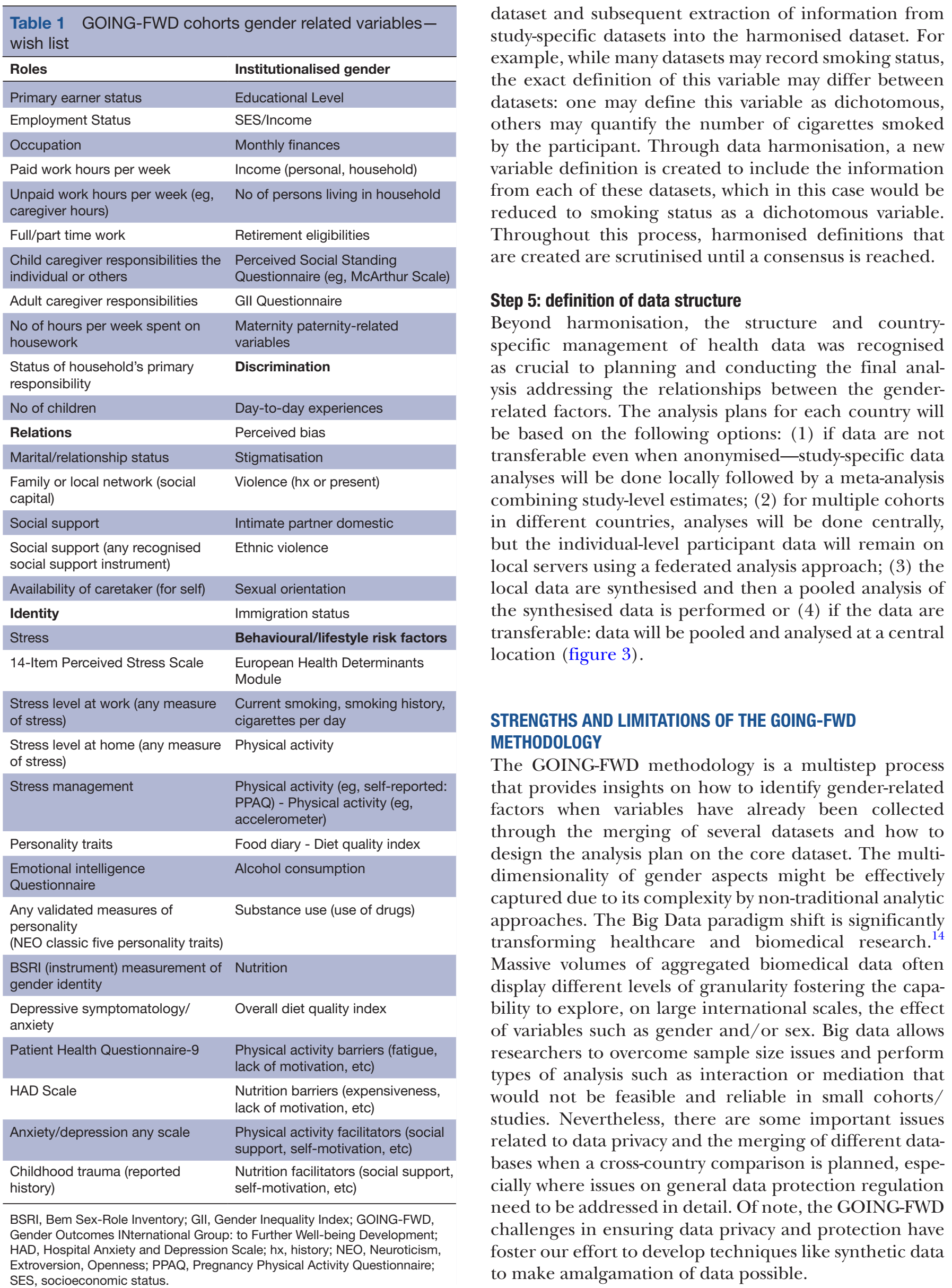




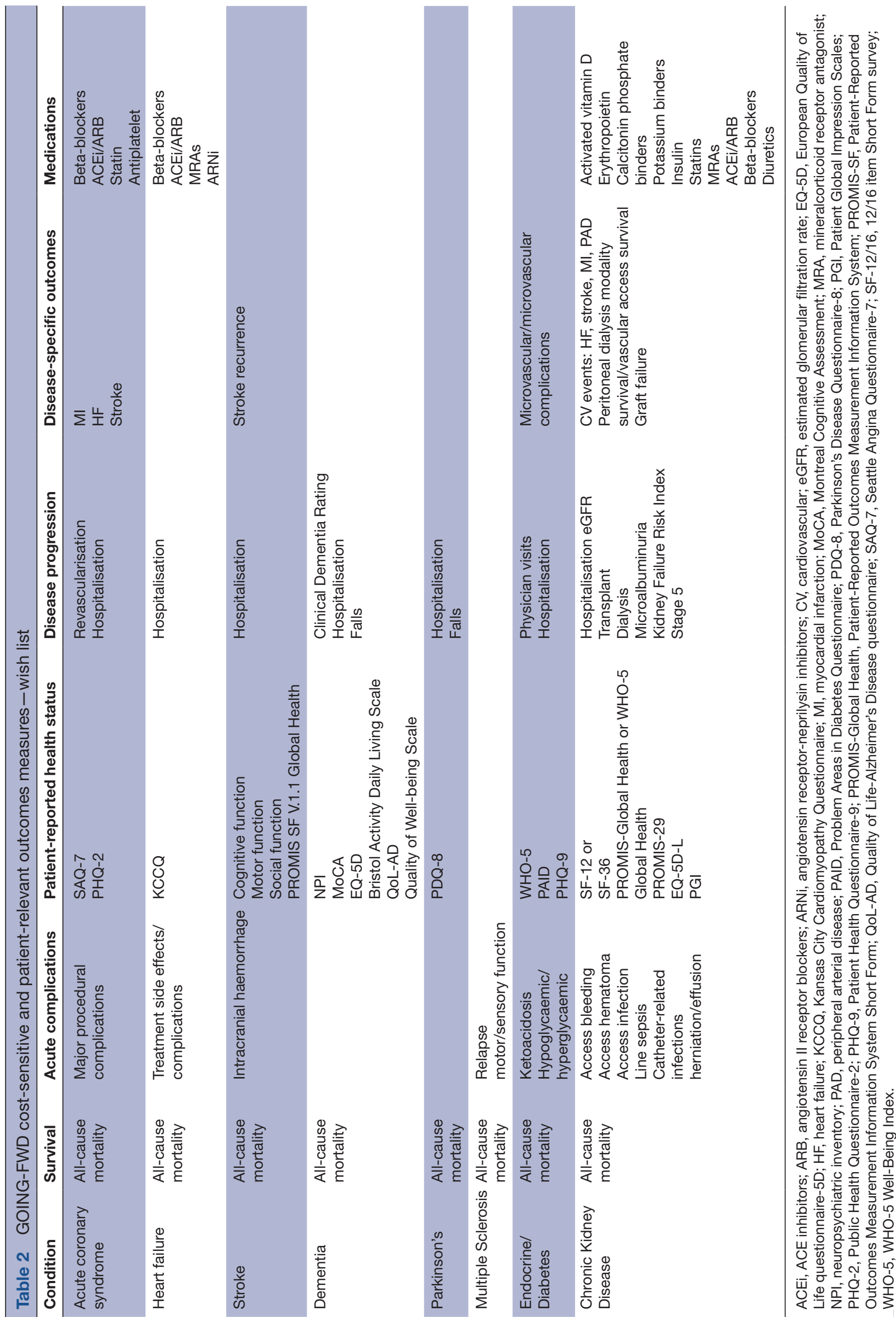




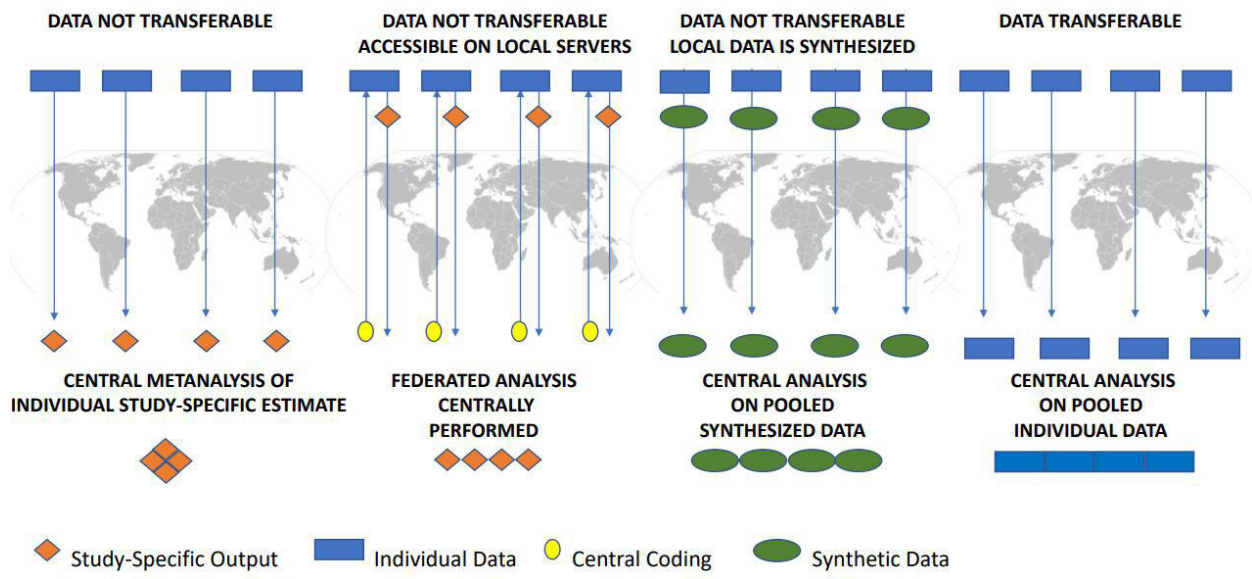

Figure 3 Data structure and potential options for analysis based on transferability of data.

\section{Strengths}

The GOING-FWD framework is a feasible methodology to foster the assessment of the gender impact on outcomes in retrospective studies. The screening of each dataset is a step that not only allows to identify the gender-related variables but also provides the rationale for selecting psycho-social factors that could be collected prospectively in the same cohort. The effort of investigating how sex and gender-related factors impact clinical and patient-related outcomes in NCDs is essential as it provides evidence for sex-tailored and gender-tailored interventions.

We have learnt that a multidisciplinary team is a prerequisite for developing such methodology including gender experts and patient partners. Patients with lived experience can contribute to understanding what is really important for a specific disease which further strengthens the concept of patient empowerment in clinical practice.

The international nature of GOING-FWD methodology highlights important considerations on the complexity of gender. Gender norms, identities and relations vary by culture, historical era, ethnicity, socioeconomic status, geographical location and other factors. We expect that the gender behaviours and attitudes captured by our variables may differ among women, men, and gender diverse individuals as well as between these groups. Gender norms also change overtime and across countries. Furthermore, everyone over time can be exposed to different degree of any gender-related factors. Impactful findings generated by retrospective analyses ca stimulate the scientific community to conduct a prospective collection on gender-factors in the design of future studies. Therefore, as researchers, we need to recognise the dynamic nature of integrating gender in clinical research questions and act accordingly. We also envisioned our multicountry analyses as an opportunity to capture institutional gender by including some country specific variables that are commonly available like the Gender Inequality Index (GII) developed by the United Nations Development Programme. ${ }^{15}$ The GII is a composite measure to quantify gender inequality within a country and measures opportunity costs, reproductive health empowerment and labour market participation. Another similar measure of gender is the European Institute for Gender Equality's Gender Equality Index, which includes additional details about country specific domains of health, violence against women, work, money, knowledge time and power. ${ }^{16}$ The idea is to relook and rethink on how we can gain the most from data on gender that are already available.

Finally, the GOING-FWD approach is timely and might foster inclusion of gender in understanding the COVID-19 pandemic. In fact, the global COVID-19 economic and medical crisis could be the first outbreak where sex and gender differences are recorded and taken into account by researchers and policy makers. The GOING-FWD methodology will be instrumental in exploring the impact of various gender domains on outcomes across countries.

\section{Challenges}

In developing the GOING-FWD methodology, we have faced practical challenges. First, the lack of a standardised definition of gender-related factors is perceived as an obstacle to researchers even if they are interested in the topic. The low availability of gender-related factors in retrospective studies is not surprising but this should not preclude analyses. We strongly encourage clinical and even preclinical researchers to start from what they have even if only one gender-related factor is available. Merging more datasets allows us to perform analyses that incorporate interaction and mediation given large sample sizes. Second, in the current era, data accessibility and data protection issues in international networks can represent a deal breaker in pursuing this kind of research approach. Increasingly strict data protection regulations in many jurisdictions limit the ability to share sensitive health information. This requires the application of privacy enhancing technologies to enable the necessary analyses to be performed without the transfer of personal health information. 
Finally, harmonisation is a necessary step to allow big data analysis, but it is a time - consuming process and susceptible to pitfalls related to the quality of the process and difficulties of maintenance when several databases from different countries are merged. Personnel with explicit knowledge and skills are required to perform data harmonisation from both technical (ie, computing science, mathematics) and clinical (ie, life science) perspectives.

We believe that our example of a derived "wish list"" based on selected variables offers a standardised tool that can be widely used to explore the consistency of associations with health behaviours and outcomes.

\section{Perspective and significance}

The GOING-FWD Consortium, a multidisciplinary network of Canadians and European researchers and patient partners, provides a framework that will support clinical researchers in integrating gender relevant factors in their research questions when using already collected databases hence providing solutions for the challenges that such approach poses.

\section{CONCLUSION}

In conclusion, the application of a systematic multistep approach defining gender-related variables, the use of data harmonisation and country-specific data structure models, inform the identification and inclusion of gender factors in retrospective cohort studies. Gleaning important information on gender will not only strengthen current clinical practice but will also provide a stepping-stone for sex-tailored and gender-tailored interventions and care.

\section{Author affiliations \\ ${ }^{1}$ Department of Translational Medicine, University of Ferrara, Ferrara, Italy ${ }^{2}$ Faculty of Nursing, University of Alberta, Edmonton, Alberta, Canada \\ ${ }^{3}$ Centre for Outcomes Research and Evaluation, Research Institute of the McGill University Health Centre, Montreal, Quebec, Canada \\ ${ }^{4}$ Department of Human Anatomy and Psychobiology, Universidad de Murcia, Murcia, Spain \\ ${ }^{5}$ Department of Medicine III, Division of Endocrinology, Medical University of Vienna, Vienna, Austria \\ ${ }^{6}$ Department of Clinical Science, Intervention and Technology (CLINTEC), Karolinska Institutet, Stockholm, Sweden \\ ${ }^{7}$ Section for Renal Medicine, Karolinska University Hospital, Stockholm, Sweden ${ }^{8}$ Electronic Health Information Laboratory, Children's Hospital of Eastern Ontario Research Institute, Ottawa, Ontario, Canada \\ ${ }^{9}$ School of Epidemiology and Public Health, Faculty of Medicine, University of Ottawa, Ottawa, Ontario, Canada \\ ${ }^{10}$ Replica Analytics Ltd, Ottawa, Ontario, Canada \\ ${ }^{11}$ Department of Medicine, Divisions of Clinical Epidemiology and General Internal Medicine, McGill University, Montreal, Quebec, Canada}

Collaborators GOING-FWD Collaborators: Karin H. Humphries; Monica Parry; Ruth Sapir-Pichhadze; Michal Abrahamowicz; Simon Bacon; Peter Klimek; Jennifer Fishman, Vera Regitz-Zagrosek; Londa Schiebinger; Carole Clair; Rachel P. Dryer; Christina P. Tadiri; Zahra Azizi; Rubee Dev; Pouria Alipour; Uri Bender; Sabeena Jalal; Alexia Della Vecchia; Jovana Stojanovic; Salima Hemani; Heather Burnside Carola Deschinger; Juergen Harreiter; Simon D. Lindner; Teresa Gisinger; Giulia Tosti; Claudia Tucci; Giulio F. Romiti; Agnè Laučytè-Cibulskiene; Liam Ward; Leah Muñoz; Raquel Gomez De Leon; Ana Maria Lucas; Sonia Gayoso; Raúl Nieto; Maria Sanchez; Sandra Amador; Cristina Rochel; Donna Hart; Nicole Hartman/
Nickerson; Angie Fullerton/MacCaul; Jeanette Smith; Myra Lefkowitz; Ann Keir; Kyle Warkentin; Rachael Manion. GOING-FWD Co-Principal Investigators: Louise Pilote, McGill University Health Center and McGill University, Canada; Colleen M. Norris, University of Alberta, Canada; Valeria Raparelli, University of Ferrara, Italy. GOING-FWD Site Principal Investigators: Alexandra Kautzky-Willer, Medical University of Vienna, Austria; Karolina Kublickiene, Karolinska Institutet, Sweden; Maria Trinidad Herrero, Universidad de Murcia, Spain. GOING-FWD Co-Investigators: Karin H. Humphries, University of British Columbia, Canada; Monica Parry, Lawrence S. Bloomberg Faculty of Nursing, Canada; Ruth Sapir-Pichhadze, McGill University Health Center and McGill University, Canada; Michal Abrahamowicz, McGill University Health Center and McGill University, Canada; Khaled El Emam, University of Ottawa, Canada; Simon Bacon, Concordia University, Canada; Peter Klimek, Medical University of Vienna, Austria; Jennifer Fishman, McGill University, Canada. GOING-FWD Scientific Advisory Committee: Vera Regitz-Zagrosek, Charité, University Medicine Berlin, German and University Hospital Zürich, University of Zürich, Switzerland; Londa Schiebinger, Stanford University, USA; Carole Clair, University of Lausanne, Switzerland; Rachel P. Dryer, Yale University, USA. GOING-FWD Early Career Researchers: Christina P. Tadiri, McGill University Health Center and McGill University, Canada; Zahra Azizi, McGill University Health Center and McGill University, Canada; Rubee Dev, University of Alberta, Faculty of Nursing, Canada; Pouria Alipour, McGill University Health Center and McGill University, Canada; Uri Bender, McGill University Health Center and McGill University, Canada; Sabeena Jalal, McGill University Health Center and McGill University, Canada; Alexia Della Vecchia, McGill University Health Center and McGill University, Canada; Jovana Stojanovic, Concordia University, Canada; Salima Hemani, Lawrence S. Bloomberg Faculty of Nursing, Canada; Heather Burnside, Lawrence S. Bloomberg Faculty of Nursing, Canada; Carola Deschinger, Medical University of Vienna, Austria; Juergen Harreiter, Medical University of Vienna, Austria; Simon D. Lindner, Medical University of Vienna, Austria; Teresa Gisinger, Medical University of Vienna, Austria; Giulia Tosti, Sapienza University of Rome, Italy; Claudia Tucci, Sapienza University of Rome, Italy; Giulio Francesco Romiti, Sapienza University of Rome, Italy; Agnẻ Laučytè-Cibulskiene, Karolinska Institutet, Sweden; Liam Ward, Karolinska Institutet, Sweden; Leah Muñoz, Karolinska Institutet, Sweden; Raquel Gomez De Leon, Universidad de Murcia, Spain; Ana Maria Lucas, Universidad de Murcia, Spain; Sonia Gayoso, Universidad de Murcia, Spain; Raúl Nieto, Universidad de Murcia, Spain; Maria Sanchez, Universidad de Murcia, Spain; Sandra Amador, Universidad de Murcia, Spain; Cristina Rochel, Universidad de Murcia, Spain.GOING-FWD Patient Partners: Donna Hart, Ontario, Canada; Nicole Hartman/Nickerson, Nova Scotia, Canada; Angie Fullerton/MacCaul, Prince Edward Island, Canada; Jeanette Smith, Ontario, Canada; Myra Lefkowitz, Ontario, Canada; Ann Keir, British Columbia, Canada; Kyle Warkentin (caregiver), British Columbia, Canada; Rachael Manion, Ontario, Canada.

Contributors VR: conception, design, interpretation of data, drafting of the manuscript and revision; CMN: conception, design, interpretation of data, critical revision; UB: acquisition and analysis; MTH, design, interpretation of data, revision of the draft; AKW: design, interpretation of data, revision of the draft; KK: design, interpretation of data, revision of the draft; KEE: analysis and development of new IT solution, synthetic data; LP: conception, design, interpretation of data, drafting of the manuscript and revision; GOING-FWD Consortium: design contribution, acquisition of data and revision of the draft. All authors read and approved the final manuscript.

Funding The GOING-FWD Consortium is funded by the GENDER-NET Plus ERANET Initiative (Project Ref. Number: GNP-78): The Canadian Institutes of Health Research (GNP-161904), La Caixa Foundation (LCF/PR/DE18/52010001), The Swedish Research Council (2018-00932) and The Austrian Science Fund (FWF, I 4209). VR was funded by the Scientific Independence of Young Researcher Program of the Italian Ministry of University, Education and Research (RBSI14HNVT).

Competing interests VR, CNM, UB, MTH, AKW, KK, and LP have nothing to disclose; KEE is co-founder, director, and investor in Replica Analytics Ltd, a CHEO Research Institute / University of Ottawa spinoff company that develops data synthesis software.

Patient consent for publication Not required.

Ethics approval Ethics approval for the project was obtained from the coordinator centre at McGill University, Canada (2020-5452).

Provenance and peer review Not commissioned; externally peer reviewed.

Open access This is an open access article distributed in accordance with the Creative Commons Attribution Non Commercial (CC BY-NC 4.0) license, which permits others to distribute, remix, adapt, build upon this work non-commercially, and license their derivative works on different terms, provided the original work is 
properly cited, appropriate credit is given, any changes made indicated, and the use is non-commercial. See: http://creativecommons.org/licenses/by-nc/4.0/.

\section{REFERENCES}

1 CIHR, Online Training Modules. Integrating Sex \& Gender in Health Research, 2019. Available: https://cihr-irsc.gc.ca/e/49347.html [Accessed 23 Apr 2020].

2 Norris CM, Murray JW, Triplett LS, et al. Gender roles in persistent sex differences in health-related quality-of-life outcomes of patients with coronary artery disease. Gend Med 2010;7:330-9.

3 Weber AM, Cislaghi B, Meausoone V, et al. Gender norms and health: insights from global survey data. Lancet 2019;393:2455-68.

4 , Forouzanfar MH, Alexander L, et al, GBD 2013 Risk Factors Collaborators. Global, regional, and national comparative risk assessment of 79 behavioural, environmental and occupational, and metabolic risks or clusters of risks in 188 countries, 1990-2013: a systematic analysis for the global burden of disease study 2013. Lancet 2015;386:2287-323.

5 Bartz D, Chitnis T, Kaiser UB, et al. Clinical advances in sex- and Gender-Informed medicine to improve the health of all: a review. JAMA Intern Med 2020;180:574-83.

6 Vaidya V, Partha G, Karmakar M. Gender differences in utilization of preventive care services in the United States. J Womens Health 2012;21:140-5.
7 Tannenbaum C, Ellis RP, Eyssel F, et al. Sex and gender analysis improves science and engineering. Nature 2019;575:137-46.

8 Pelletier R, Ditto B, Pilote L. A composite measure of gender and its association with risk factors in patients with premature acute coronary syndrome. Psychosom Med 2015;77:517-26.

9 Pelletier R, Khan NA, Cox J, et al. Sex versus gender-related characteristics: which predicts outcome after acute coronary syndrome in the young? J Am Coll Cardiol 2016;67:127-35.

10 Norris CM, Johnson NL, Hardwicke-Brown E, et al. The contribution of gender to apparent sex differences in health status among patients with coronary artery disease. $J$ Womens Health 2017:26:50-7.

11 Johnson JL, Greaves L, Repta R. Better science with sex and gender: facilitating the use of a sex and gender-based analysis in health research. Int J Equity Health 2009;8:14.

12 International Consortium for health outcomes measurement, 2020. Available: https://www.ichom.org/ [Accessed 22 May 2020].

13 Fortier I, Raina P, Van den Heuvel ER, et al. Maelstrom research guidelines for rigorous retrospective data harmonization. Int $\mathrm{J}$ Epidemiol 2017;46:103-5.

14 Obermeyer Z, Emanuel EJ. Predicting the Future - Big Data, Machine Learning, and Clinical Medicine. N Engl J Med 2016;375:1216-9.

15 Gender inequality index (GII), 2020. Available: http://hdr.undp.org/en/ content/gender-inequality-index-gii [Accessed 22 May 2020].

16 Gender equality index, 2020. Available: https://eige.europa.eu/ gender-equality-index/2019 [Accessed 22 May 2020]. 